\title{
Optically Stimulated Luminescence Materials for Wide-Spectrum Neutron Measurements at CERN
}

\author{
F. Ravotti, Student Member, IEEE, M. Glaser, K. Idri, J.-R. Vaillé, H. Prevost, and L. Dusseau, Senior Member, IEEE
}

\begin{abstract}
For the first time, Optically Stimulated Luminescent (OSL) materials with enhanced neutron-sensitivity have been irradiated. The neutron irradiations were carried out in the accelerator-like environment of the IRRAD2 facility at CERN, Geneva, Switzerland and in the TRIGA reactor at JSI, Ljubljana, Slovenia. The results show the possibility to measure with good accuracy the thermal and the fast components of these two different neutron spectra by means of OSL materials doped with Boron or mixed with Polyethylene. The applicability of this technology in the measurement of the neutron components in the complex radiation environment expected at CERN LHC experiments is therefore demonstrated.
\end{abstract}

Index Terms-Neutron dosimetry, optically stimulated luminescent (OSL) dosimetry, particle beams.

\section{INTRODUCTION}

$\mathbf{T}$ HE radiation environment expected in the future CERN Large Hadron Collider (LHC) experiments will be composed of neutrons, photons and charged hadrons. This complex field, which has been simulated by Monte Carlo codes [1], [2], is due to particles generated by the proton-proton collisions and reaction products of these particles with the sub-detector materials of the experiment itself. Neutrons, becoming dominant at large radii with respect to the interaction point, are a matter of concern due to radiation damage to all exposed particle detectors and electronic components [3]. For example, in the EndCap electromagnetic calorimeter of CMS as well as in the innermost barrel layer of the ATLAS liquid argon calorimeter, an annual $1 \mathrm{MeV}$ equivalent neutron fluence of more than $10^{13} \mathrm{~cm}^{-2}$ is expected.

An integrated sensor based on different dosimetric technologies, including Optically Stimulated Luminescence (OSL) materials, RadFETs and $p-i-n$ diodes is currently under development for the radiation monitoring of the LHC experiments [4]. An overview of this work was already given elsewhere [5], [6]. The present paper details a mandatory step in the OSL qualification process: the evaluation of neutron sensitivity and neutron energy dependence.

OSL materials as radiation dosimeters for medical and space applications have been studied extensively [7], [8]. For

Manuscript received September 22, 2004; revised April 11, 2005.

F. Ravotti is with the Department TS-LEA-RAD, CERN, Geneva, 23, CH-1211, Switzerland, and also with CEM2, Université Montpellier II, 34095 Montpellier Cedex 5, France (e-mail: federico.ravotti@ cern.ch).

M. Glaser is with the Department PH-DT2-SD, CERN, Geneva, 23, CH-1211, Switzerland (e-mail: maurice.glaser@cern.ch).

K. Idri, J.-R. Vaillé, H. Prevost, and L. Dusseau are with the CEM2, Université Montpellier II, 34095 Montpellier Cedex 5, France (e-mail: dusseau@cem2.univ-montp2.fr).

Digital Object Identifier 10.1109/TNS.2005.855821
High-Energy Physics (HEP) applications, successful experiments were carried out to measure the dose delivered by different charged particles [9]. Since there is a lack of on-line neutron dosimeters for HEP applications, especially for thermal neutron energies, the possibility to make OSL material sensitive to neutrons is a very attractive prospect. However, the OSL response to neutron irradiation has still to be studied, as a prerequisite to a possible use in the CERN radiation environments.

A first irradiation campaign was thus carried out on special OSL materials with an enhanced sensitivity to different neutron energies. The OSL standard materials were doped with Boron or mixed with polyethylene to increase their sensitivity to thermal and fast neutrons respectively. The samples, prepared from the Electronic and Radiations Research Laboratory of the University Montpellier II, France, were irradiated in the IRRAD2 Facility at the CERN Proton-Synchrotron (PS) [10] and at the TRIGA reactor facility at the Jozef Stefan Institute (JSI) of Ljubljana, Slovenia [11].

The principles of OSL dosimeters used for neutron detection are reviewed in Section II. In Section III, the experiments in the two neutron facilities are briefly described. In Section IV, the results are summarized and discussed. Finally, our conclusions are given in Section V.

\section{REVIEW OF OSL MATERIAL AS NEUTRON DETECTOR}

The OSL materials have the property to emit visible light proportional to the received dose of radiation.

Ionizing radiation creates electron-hole pairs in the OSL material. A fraction of these carriers is trapped in energy levels located in the wide band gap of the insulator. These charges will remain trapped for a period of time depending on the activation energy of the traps and the temperature. When the energy necessary to release the charges is provided by an optical stimulation, a radiative recombination may be observed. Quantifying the amount of the emitted light makes it possible to evaluate the dose [12].

Neutrons do not produce direct ionization in materials; however, a little amount of ionizing energy is deposited via secondary particles generated by neutron interactions. The total neutron cross sections for the elements constituting the used OSL material are in the order of a few barns for neutron energies ranging from thermal to several $\mathrm{MeV}$. The resulting energy deposition is usually not sufficient to generate a measurable quantity of electron-hole pairs in pure OSL.

For this reason, different additives were mixed with the standard OSL material that consists of doped Strontium Sulfide (SrS). In particular: Boron (B) was added to increase the OSL sensitivity to thermal neutrons and polyethylene (PE) was added for fast neutron detection. 


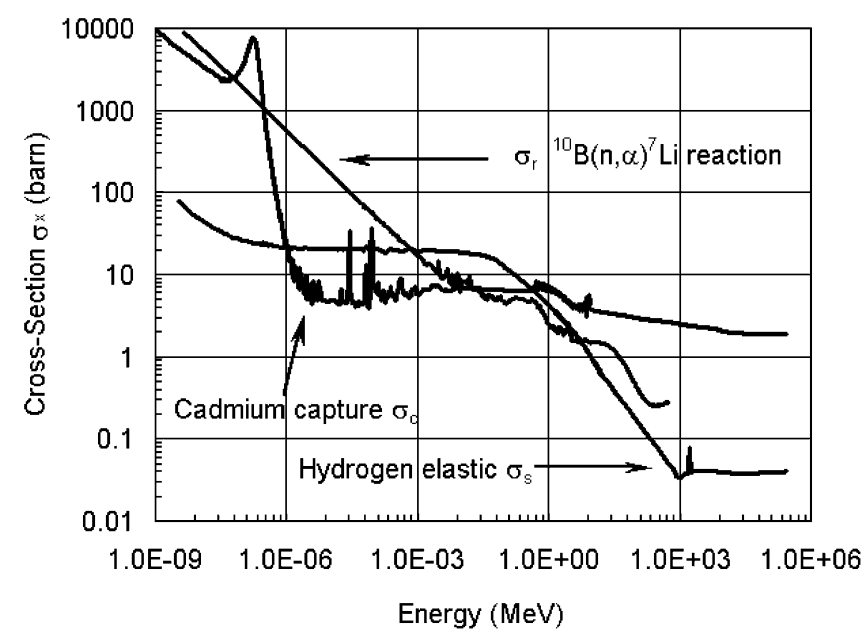

Fig. 1. Plot of the neutron reaction cross sections of interest for this work [13].

Boron is responsible for thermal neutron capture via the nuclear reaction

$$
{ }^{10} \mathrm{~B}+n \rightarrow{ }^{7} \mathrm{Li}+\alpha+\gamma+2.3 \mathrm{MeV} .
$$

This reaction has a very large cross-section $\sigma_{r}=3840$ barns for thermal energies that decreases down to a few barns for energies above some tens of $\mathrm{keV}$ as shown in Fig. 1 [13]. The alpha particles and the lithium ions generated by the neutron capture lose their energy via ionization in the sensitive SrS material. The ranges of the two reaction products in the $\mathrm{SrS}$ were calculated with the SRIM software [14]. Taking into account that the $2.3 \mathrm{MeV}$ released in the reaction contributes to the kinetic energy of the lithium ion $(0.83 \mathrm{MeV})$ and the alpha particle $(1.47 \mathrm{MeV})$, ranges of 2 and $4.2 \mu \mathrm{m}$ in SrS were calculated respectively. These short path-lengths guarantee that all captured thermal neutrons deposit the full ionization energy in the OSL material.

$\mathrm{PE}$ with its high content of hydrogen is the preferred material to detect fast neutrons because a colliding particle can transfer from none to all its energy to the proton in one elastic collision. The average recoil energy will be half of the initial neutron energy. Hydrogen has a cross section $\sigma_{s}$ of the order of tens of barns for epi-thermal neutrons that falls to 4 barns for the energy of $1 \mathrm{MeV}$. It further decreases to approximately 1 barn when the neutron energy reaches $8 \mathrm{MeV}$ as shown in Fig. 1. The recoil proton will move through the sensitive material with a path length depending on the energy transferred to it by the incident neutron.

\section{EXPERIMENTS}

\section{A. Samples Manufacturing}

The pure OSL material and three compounds of OSL material doped with Boron and mixed with polyethylene were prepared and irradiated to different neutron fluences.

The four different types of OSL samples are shown in Fig. 2. They have a size of $5 \times 5 \mathrm{~mm}^{2}$ and various thicknesses. In particular, the used materials had the following characteristics.

1) OSL standard material (OSL pure): Specimens obtained from a $100 \mu \mathrm{m}$ thick layer of SrS silk-screen printed on a Kapton foil.

2) OSL doped with Boron $(\mathrm{OSL}+B)$ : Specimens obtained from a $100 \mu \mathrm{m}$ thick layer of SrS, $1 \%$-weight Boron

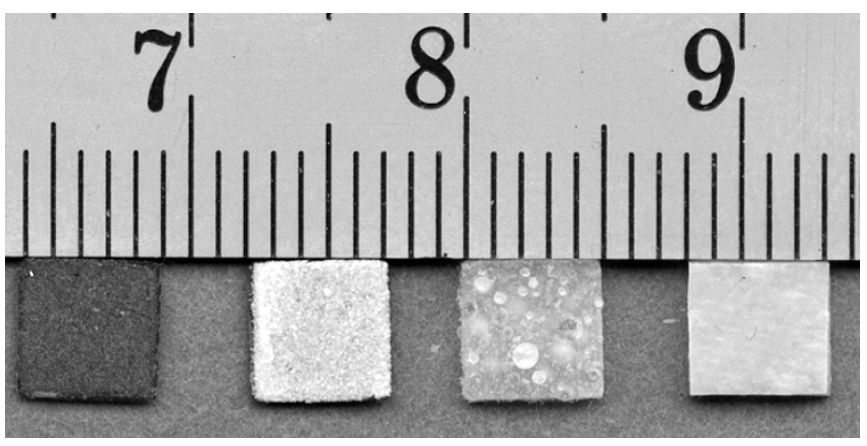

Fig. 2. OSL materials from left to right: Pure OSL material, OSL doped with Boron, OSL mixed with Polyethylene, and OSL mixed with Polyethylene and Boron.

doped, silk-screen printed on a Kapton foil. Boron was introduced in the OSL material through sintering and subsequent grinding procedures, ensuring the homogeneity of the obtained samples.

3) OSL mixed with Polyethylene (OSL + PE): Specimens gained from a bigger block in which the SrS was melted with polyethylene. The thicknesses of the samples were of a few mm.

4) OSL mixed with PE and Boron (OSL $+\mathrm{PE}+B)$ : OSL specimens containing both, PE and Boron. The thicknesses of these samples were also of a few $\mathrm{mm}$.

Of each type of OSL material, three to five pieces were used in order to prove the reproducibility of the results. They were packaged and irradiated in few micron-thick aluminum foils in order to protect them from light. Before irradiation, the samples were heated to $80^{\circ} \mathrm{C}$ for $24 \mathrm{~h}$ to anneal the charge in the OSL trap levels (i.e., to clear the dosimetric material).

\section{B. CERN IRRAD2 Neutron Facility}

The irradiation in the facility IRRAD2 at the CERN-PS accelerator is performed in a cavity with secondary particles produced by the primary $24 \mathrm{GeV} / \mathrm{c}$ proton beam after crossing a thick beam dump consisting of carbon and iron blocks [10].

A motorized shuttle system allows the transport of samples from the counting room to the irradiation cavity in which a broad spectrum of neutrons is produced as shown in Fig. 3 [15].

Together with neutrons, high-energy hadrons and gamma rays are produced. Gamma rays represent a contamination that is in the order of $10 \%-20 \%$ with respect to the neutron component, depending on the operation conditions.

With the remote shuttle system, it is possible to set the position of the samples with respect to the beam axis. At positions far from the beam axis, the ratio of charged hadrons in the $\mathrm{GeV}$ energy range to neutrons can be considerably reduced and it is thus possible to perform irradiations in an almost pure neutron environment. The OSL samples were thus placed at $50 \mathrm{~cm}$ from beam axis and exposed to four different $1 \mathrm{MeV}$ neutron equivalent fluences, reaching a maximum value of $1.6 \times 10^{12} \mathrm{~cm}^{-2}$. In this facility at $50 \mathrm{~cm}$ a hardness factor $k=0.67$ has been calculated.

\section{TRIGA Reactor Ljubljana Neutron Facility}

The Reactor Research Centre at the Jozef Stefan Institute (JSI) in Ljubljana has an experimental nuclear reactor of type TRIGA [11]. 


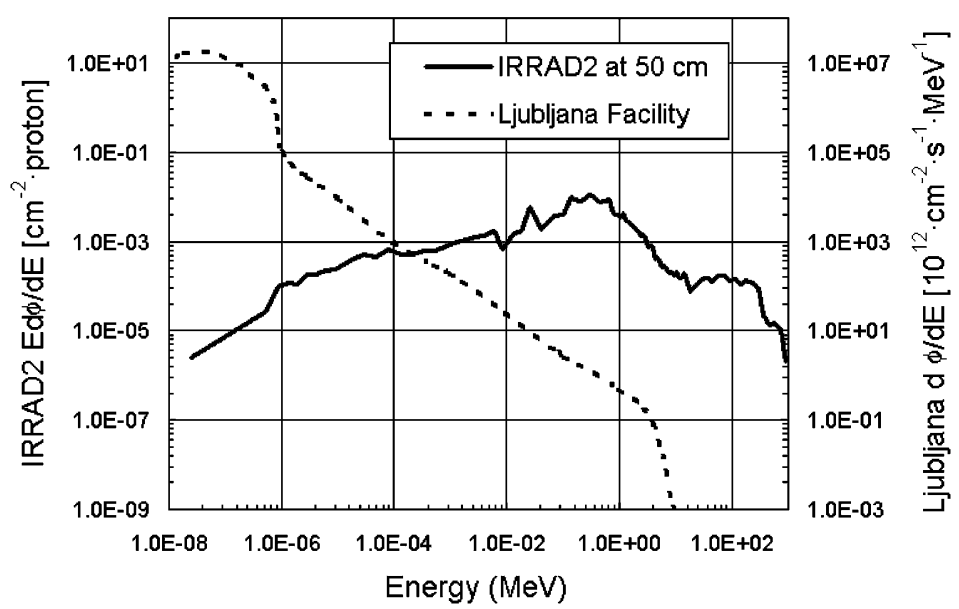

Fig. 3. Neutron spectrum in the IRRAD2 Facility at $50 \mathrm{~cm}$ from beam axis normalized to one impinging proton (continuous line). Ljubljana Reactor neutron spectrum as measured from activation foils (dotted line).

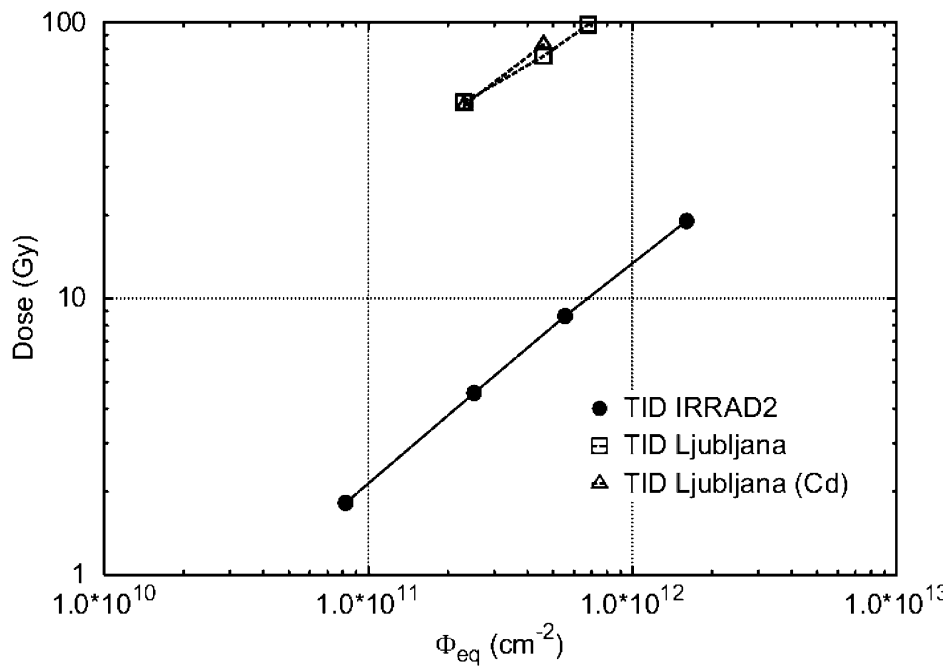

Fig. 4. TID measured in both irradiation facilities by means of Gafchromic sensitive film HD-810.

The neutron spectrum of this facility, determined by measuring the activation of foils of different materials, is shown in Fig. 3 [16].

Irradiations were performed placing the OSL samples into the reactor core through an irradiation tube that occupies a fuel rod position and enters the reactor core from above. The neutron flux delivered to the samples is linearly proportional to the reactor operating power.

A first series of OSLs was placed in the core, and exposed to three different $1 \mathrm{MeV}$ neutron equivalent fluences, reaching a maximum value of $6.9 \times 10^{11} \mathrm{~cm}^{-2}$. In this facility a hardness factor $k=0.90$ has been determined. A second series was shielded by means of a $1.0 \mathrm{~mm}$ thick Cadmium $(\mathrm{Cd})$ foil in order to irradiate the OSLs without the thermal neutron component of the reactor spectrum (see $\sigma_{c}$ behavior in Fig. 1). The Cd thickness was chosen according to the thermal neutron mean-freepath that is of about $100 \mu \mathrm{m}$.

\section{Dosimetric Measurements During Irradiations}

Gafchromic (Gafchromic is a registered trademark of ISP Corporation) Dosimetric Films HD-810 [17] and silicon detectors [18] were used in both facilities to measure the Total Ionizing Dose (TID) and the neutron fluence respectively.
The film Gafchromic HD-810 develops a characteristic blue color upon exposure to ionizing radiation and becomes progressively darker in proportion to the absorbed dose. The color change has been measured after irradiation on samples of one square centimeter by means of a transmission densitometer. The film calibration used in the present work was performed using the $24 \mathrm{GeV} / \mathrm{c}$ protons of the IRRAD1 facility at CERN [19].

Silicon detectors are reverse biased $p-i-n$ diodes with a very high resistivity $n$-type bulk. Neutron irradiation of such devices will produce generation/recombination centers in the silicon bulk leading to a fluence proportional increase of the leakage current when the device is kept fully depleted [20]. With this technique, it is possible to obtain fluence measurements with an accuracy of $\pm 10 \%$.

In the CERN facility, silicon detectors and films were exposed together with the OSLs, while only films were used during Ljubljana irradiations, since the neutron flux in the reactor was previously calibrated.

Fig. 4 shows the results of the TID measurements performed in the two facilities. In the Ljubljana reactor, the TID measured for the samples irradiated in a Cd shielding (triangular markers) is presented separately from the unshielded samples (square markers). 


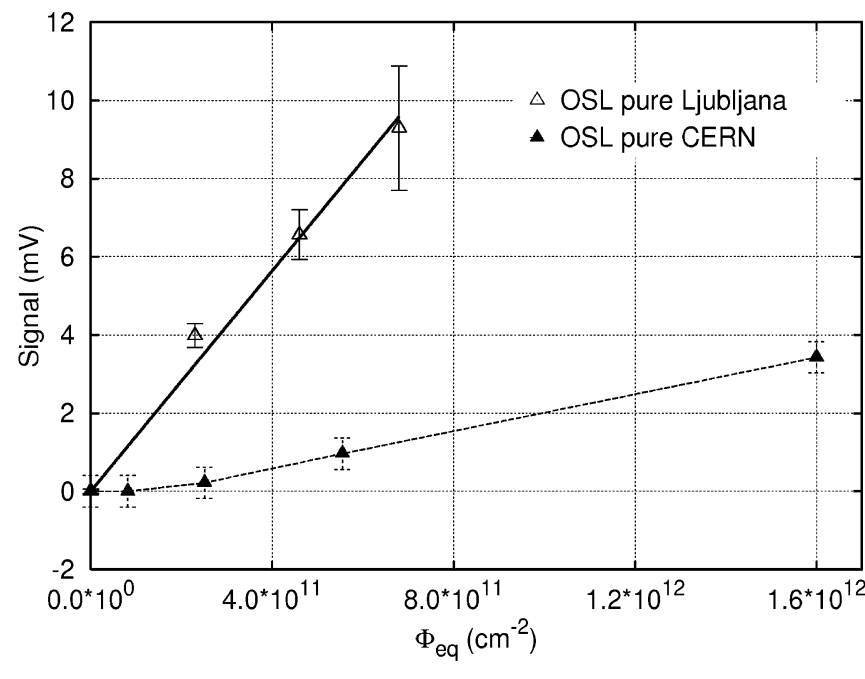

Fig. 5. Pure OSL material response after irradiation at CERN (filled markers) and Ljubljana (empty markers).

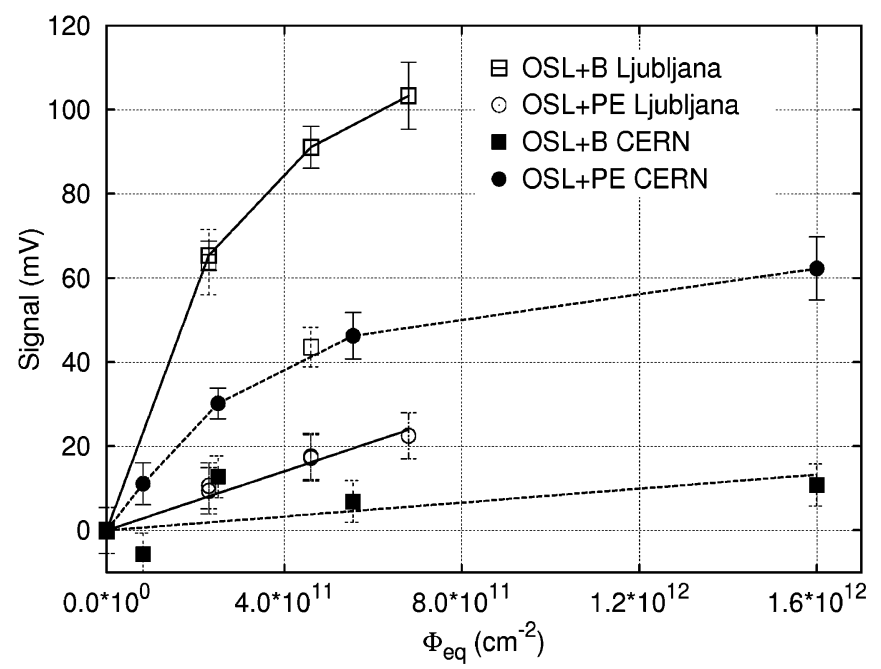

Fig. 6. OSL $+B$ and OSL $+\mathrm{PE}$ material response after irradiation at CERN (filled markers) and Ljubljana (empty markers).

Due to the high gamma background in the Ljubljana reactor, the TID results are about a factor of ten higher than in the CERN facility.

\section{RESUlTS AND Discussion}

All samples were stored at room temperature and measured a few weeks after irradiation. From previous experiences carried out on pure SrS material, the fading of OSLs was expected to be stabilized at the readout time [21]. The OSL reader used in this work is an infrared LED focused on the irradiated specimen that is positioned in a samples holder in a light-tight box. The emitted luminescent signal is collected by means of a photomultiplier, then digitalized and recorded by a PC connected to the test bench via a RS-232 interface [7], [9].

The signal (voltage) measured with the above described testbench has been normalized to the pure OSL sample's weight in order to take into account the different sample's volume. The so treated signals expressed in $\mathrm{mV}$ have been plotted versus the
TABLE I

RELATIVE SENSITIVITY OF OSLS TO NEUTRONS

\begin{tabular}{ccc}
\hline & OSL+B / OSL pure & OSL+PE / OSL pure \\
\hline$C E R N$ & 4 & 25 \\
\hline Ljubljana & 20.1 & 2.5 \\
\hline
\end{tabular}

TABLE II

SPECTRA COMPOSITION IN THE NEUTRON FACILITIES

\begin{tabular}{ccccc}
\hline & $\begin{array}{c}\text { Thermal } \\
(<1 \mathrm{eV})\end{array}$ & $\begin{array}{c}\text { Epi-Thermal } \\
(1 \mathrm{eV}-50 \mathrm{keV})\end{array}$ & $\begin{array}{c}\text { Fast } \\
(50 \mathrm{keV}-4 \mathrm{MeV})\end{array}$ & $\begin{array}{c}\text { High- } \\
\text { Energy } \\
(>4 \mathrm{MeV})\end{array}$ \\
\hline CERN & $0.2 \%$ & $15.3 \%$ & $81.3 \%$ & $3.2 \%$ \\
\hline Ljubljana & $52 \%$ & $28 \%$ & $17 \%$ & $3 \%$ \\
\hline
\end{tabular}

$1 \mathrm{MeV}$ neutron equivalent fluence, and are presented in Figs. 5 and 6.

After both irradiations, the signal achieved with the OSL pure material is the smallest recorded in our tests, and is less than $10 \mathrm{mV}$ as shown in Fig. 5. In the CERN facility (triangular filled markers), for values of $1 \mathrm{MeV}$ neutron equivalent fluence below $1.0 \times 10^{11} \mathrm{~cm}^{-2}$, the signal was lower than the noise level since the gain of the read out system was set to measure high doses. For the Ljubljana facility (triangular empty markers) the signal is a factor of ten higher with respect to the one measured at CERN. This is in good agreement with the TID measurements presented in Fig. 4 of Section III-D. As expected, the OSL pure material measured thus the ionizing component of the field and is essentially insensitive to neutrons.

Fig. 6 shows the results obtained for the OSL $+B$ and OSL + PE materials. In the higher fluence range saturation is observed. This is also expected for the OSL pure materials at fluences which are exceeding the range presented in Fig. 5. The corresponding experimental data have been presented in [5]. For a comparison between the neutron-sensitive and the pure material linear fits have been applied to the data measured in the low fluence range. A 20 times increased sensitivity has been found in this way as presented in Table I.

In the Ljubljana facility (continuous lines in Fig. 6) for an equivalent neutron fluence of $2 \times 10^{11} \mathrm{~cm}^{-2}$, the measured signals from the OSL $+B$ (square empty markers) and OSL $+\mathrm{PE}$ (round empty markers) samples represent $85 \%$ and $15 \%$, respectively, of the total neutron signal. At CERN (dotted lines in Fig. 6) and for the same neutron fluence, the signals obtained from the OSL + PE samples (round filled markers) represent more than $93 \%$ of the neutrons signal, as the OSL $+B$ (square solid markers) samples gave only a little signal.

All the above results are in good agreement with the spectra composition of the two neutron environments as shown in Fig. 3 of Section III-B and summarized in Table II.

The strongly fluctuating signal of the OSL $+B$ samples obtained at the CERN facility can be explained as a difficulty in the measurement of a signal that is close to the electronic noise level of the readout apparatus.

Moreover, one of the samples irradiated in Ljubljana also shows a reduced response in the same fluence range (see the square empty markers at $4.6 \times 10^{11} \mathrm{~cm}^{-2}$ of Fig. 6). Again, an experimental problem is suspected, which requires crosschecking in a forthcoming irradiation. However, at this moment 


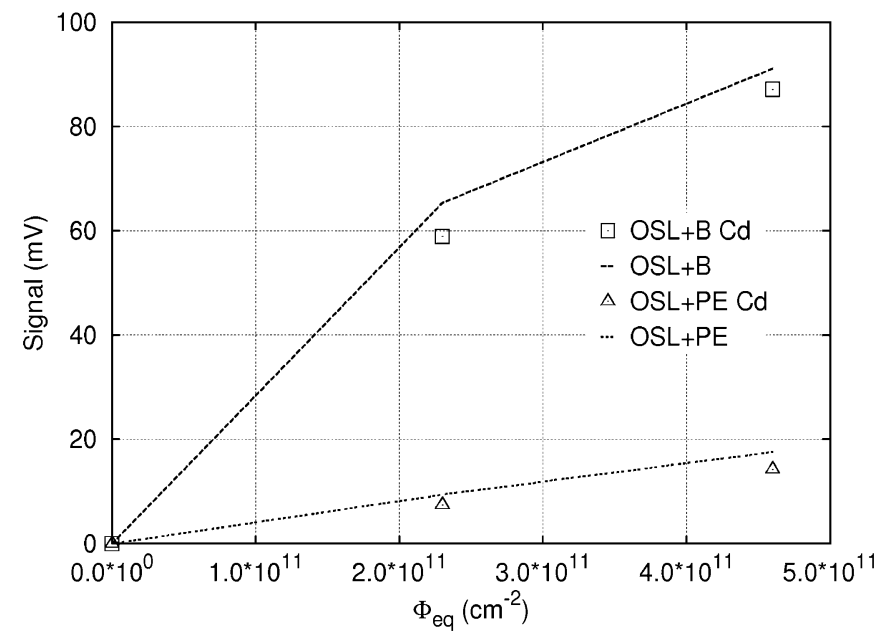

Fig. 7. Series of samples irradiated in the Ljubljana reactor while a Cd shielding suppressed the thermal neutron component of the spectrum (markers). The signals measured for the samples irradiated with the whole spectrum (unshielded samples) are also shown for comparison (dashed lines).

it is not possible to exclude a possible influence of the Boron doping on the OSL's annealing behavior.

For the OSL $+\mathrm{PE}+B$ only the Ljubljana data are available. This material gave the same response as the OSL $+\mathrm{PE}$ that is already plotted in Fig. 6 . The current method to produce $\mathrm{OSL}+\mathrm{PE}+B$ specimens leads to a predominant polyethylene fraction compared to the Boron one and is likely to account for this effect.

Finally, for the Ljubljana samples, an inter-comparison of $\mathrm{OSL}+B$ and OSL $+\mathrm{PE}$ samples irradiated with and without the thermal neutron component of the spectrum (i.e., without or with Cd shielding) is presented in Fig. 7.

The shielded OSL $+B$ and OSL + PE samples show a $10 \%$ reduced response. However, according to calculations using the cross sections of Fig. 1 and the Ljubljana neutron spectra of Fig. 3, the signals coming from the Cd-shielded OSL $+B$ and OSL + PE samples were expected to be reduced by about $70 \%$ and a few percent with respect to the unshielded ones. The calculation for the OSL + PE samples take into account that recoil protons of energies $<100 \mathrm{eV}$ release a negligible amount of energy in ionization processes into the sensitive material. The $\mathrm{Cd}$ shielding should not thus have effect on the signal measured from the OSL $+\mathrm{PE}$ samples. This is also observed in the experimental data presented in Fig. 7 where the difference between the shielded and the unshielded OSL + PE samples is within the relative errors of the experiment.

However, the drastic expected reduction of the signal for the Cd-shielded OSL $+B$ samples is not observed. This could be due to the mechanical difficulties in the realization of a tight shielding using $1 \mathrm{~mm}$ thick $\mathrm{Cd}$ foil.

The maximum relative error evaluated on voltage measurement of samples of the same type exposed to the same irradiation environment (usually three to five samples used per data point, see Section III-A) over all series of irradiated OSL materials was $7.9 \%$ and $12.0 \%$ for the Ljubljana and for the CERN samples respectively, proving a good reproducibility of the measured results.
For doses exceeding the mGy range in pure ionizing environments a reproducibility of about $3 \%$ has been measured for the OSL technology [21].

One error source in the presented measurements is probably due to misalignments that can occur during the positioning of the irradiated specimens in the samples holder of the test-bench.

\section{CONCLUSION AND OUTLOOK}

For the first time the different components of wide neutron spectra have been measured by means of specially prepared OSL materials.

Results of neutron irradiations carried out in the acceleratorlike environment of the CERN-PS IRRAD2 facility and in the environment of the TRIGA reactor in Ljubljana were presented. Pure OSL material ( $\mathrm{SrS}$ ) doped with Boron or mixed with polyethylene allowed to measure the thermal and the fast components of these two completely different neutron spectra.

However, further tests in both neutron and pure ionizing radiation environments have to be performed to better understand the behavior of the samples doped with Boron, and any possible influence of the doping on the material's annealing behavior. According to Thermoluminescence and Photoluminescence measurements the optical self-absorption in neutron-sensitive as well as the OSL pure compounds can be neglected [22]; however the influence of the sample's thickness on the response will also be investigated in detail.

These results already prove that properly prepared OSLs are a promising option for the monitoring of the different neutron components in the complex radiation environments of the CERN LHC experiments.

New specimens of pure and neutron-sensitive OSLs have already been prepared. To improve the samples readout, the $O S L$ pure and OSL $+B$ materials have been deposited directly on the active surface of GaAsP visible photodiodes sealed in a TO-5 package. For fast-neutron detection the package of some OSL pure samples were then filled up with paraffin.

Further neutron irradiations over an extended fluence range will be performed. With these samples the feasibility of a "real-time" readout, as desirable for radiation monitoring in the CERN LHC experiments, will also be tested.

\section{ACKNOWLEDGMENT}

The authors would like to thank M. Moll, C. Joram, and E. Tsesmelis from CERN for clarifying discussions and valuable comments during the preparation of this paper, as well as I. Mandić and M. Mikuž, and all the staff and operators of the TRIGA reactor at the Jozef Stefan Institute of Ljubljana, Slovenia, for performing the OSL irradiations. They would also like to thank the CERN TS-LEA group for their general support.

\section{REFERENCES}

[1] I. Dawson and C. Buttar, "The radiation environment in the ATLAS inner detector," Nucl. Instrm. Meth. A, vol. 453, pp. 461-467, 2000.

[2] M. Huhtinen, "Radiation Environment Simulations for the CMS Detector," CERN, CMS Tech. Note 95-198, 1995.

[3] A. G. Holmes-Siedle and L. Adams, Handbook of Radiation Effects, 2nd ed. Oxford, U.K.: Oxford Univ. Press, 2002. 
[4] LHC Experiment Radiation Monitoring Working Group. RADMON. [Online]. Available: http://cern.ch/lhc-expt-radmon/

[5] F. Ravotti, M. Glaser, M. Moll, K. Idri, J.-R. Vaille, H. Prevost, and L. Dusseau, "Conception of an integrated sensor for the radiation monitoring of the CMS experiment at the large hadron collider," IEEE Trans. Nucl. Sci., vol. 51, no. 6, pp. 3642-3648, Dec. 2004.

[6] F. Ravotti and M. Glaser, "A Study of the Response of Solid-State Dosimeters to be Used for the Measurement of the Radiation Environment of the CMS Experiment at the LHC," CERN, Tech. Note CERN-EST-LEA/2003-03, 2003.

[7] G. Polge et al., "Applications to radiotherapy of a dose-mapping system based on the optically stimulated luminescence," presented at the IEEE Medical Imaging Conf., Lyon, France, Oct. 2-6, 2000.

[8] L. Dusseau, G. Polge, S. Matias, J. R. Vaillé, R. Germanicus, R. Broadhead, B. Camanzi, M. Glaser, F. Saigne, J. Fesquet, and J. Gasiot, "Highenergy particle irradiation of optically stimulated luminescent films at CERN,” IEEE Trans. Nucl. Sci., vol. 48, no. 6, pp. 2056-2066, Dec. 2001.

[9] D. Plattard, G. Ranchoux, L. Dusseau, G. Polge, J.-R. Vaille, J. Gasiot, J. Fesquet, R. Ecoffet, and N. Iborra-Brassart, "Characterization of an integrated sensor using optically stimulate luminescence for in-flight dosimetry," IEEE Trans. Nucl. Sci., vol. 49, no. 3, pp. 1322-1326, Jun. 2002 .

[10] M. Glaser, L. Durieu, F. Lemeilleur, M. Tavlet, C. Leroy, and P. Roy, "New irradiation zones at the CERN-PS," Nucl. Instrum. Meth. A, vol. 426, pp. 72-77, 1999.

[11] M. Ravnik and R. Jeraj, "Research reactor benchmarks," Nucl. Sci. Eng., vol. 145, pp. 145-152, 2003.

[12] O. Missous, F. Loup, J. Fesquet, H. Prevost, and J. Gasiot, "Optically stimulated luminescence of rare-earth doped phosphors," Eur. J. Solid State Inorg. Chem., vol. 28/s, pp. 163-166, 1991.
[13] (2003) EXFOR Library of Experimental Nuclear Reaction Data v. 1.10, IAEA Nuclear Data Service. [Online]. Available: http://www-nds.iaea.org/

[14] (2003) The Stopping and Range of Ions in Matter. SRIM. [Online] Available: http://www.srim.org

[15] M. Glaser, M. Huhtinen, F. Lemeilleur, C. Leroy, P. Roy, and M. Tavlet, "Radiation test facilities in the new PS East Hall at CERN," in Proc. IEEE RADECS, Fontevraud, France, Sep. 13-17, 1999, pp. 136-141.

[16] E. Krištof, "Characterization of neutron flux in the exposure channel F19 of the TRIGA MARK II reactor in Ljubljana," in Proc. Nuclear Energy in Central Europe Conf., Terme Catež, Slovenia, Sep. 7-10, 1998, pp. 43-48.

[17] Gafchromic Sensitive Films Datasheets. [Online]. Available: http://www.ispcorp.com

[18] M. Moll, E. Fretwurst, and G. Lindström, "Leakage current of hadron irradiated silicon detectors-Material dependence," Nucl. Instrm. Meth. A, vol. 426, pp. 87-93, 1999.

[19] M. Glaser, F. Ravotti, and M. Moll, "Dosimetry assessments in the irradiation facilities at the CERN-PS accelerator," presented at the 8th Eur. Conf. RADECS, Cap d'Adgde, France, Sep. 19-23, 2005.

[20] M. Moll, E. Fretwurst, M. Kuhnke, and G. Lindström, "Relation between microscopic defects and macroscopic changes in silicon detector properties after hadron irradiation," Nucl. Instrum. Meth. B, vol. 186, pp. 100-110, 2002.

[21] J.-R. Vaillé, "Développement, Caractérization et Durcissement d'un Dosimétre Basé Sur la Luminescence Stimulée Optiquement Destiné aux Applications Spatiales (Thesis)," Ph.D. dissertation, Dept. Electron., Univ. Montpellier II, Montpellier, France, 2003.

[22] H. Prevost, "Private Communication," University Montpellier II, Montpellier, France, 2005. 